\title{
EXPRESSION OF TYPE 1 FIMBRIAE ENHANCES VIRULENCE OF UROPATHOGENIC E. COLI BY BIOFILM FORIMATION
}

KEY WORDS: E. coli, Biofilm, Urinary Tract Infection, fimH

\section{Dr Niraj Ghanwate* \\ Dr Prashant Thakare}

Assistant Professor, Department of Microbiology, Sant Gadge Baba Amravati University, Amravati, India. *Corresponding Author

Associate Professor, Department of Biotechnology, Sant Gadge Baba Amravati University, Amravati, India.

BACKGROUND: Many bacterial infections are associated with biofilm formation. It is one of the important virulent factors of $E$. coli in urinary tract, causing recurrent and drug resistant infections. Fecal $E$. coli colonize the urethra and spread up the urinary tract to the bladder and kidney. Type 1 fimbriae are surface located adhesion organelles of E. coli that are directly associated with adherence to the urinary tract. The present study was aimed to study biofilm production in E. coli isolated from urinary tract infection and to correlate it with expression of fimH gene and compare its sequences. METHOD: Total 150 urine samples were processed for isolation and identification of uropathogens. E. coli isolates were further processed for detection of biofilm by TCP method and screened for the presence of fimH gene by PCR using specific primers. The PCR products were purified and sequenced bidirectionally by Sanger dideoxy sequencing system using ABI 3500 Genetic analyzer.

RESULTS: From the total 98 urine samples with significant bacteriuria, $77 \mathrm{E}$. coli were isolated out of which, 40 were positive for in vitro biofilm production. Among them 11 were classified as strong biofilm producers and 29 as moderate. The fimH gene from E. coli isolates was amplified using specific primers and appeared as a band of about 508bp on agarose gel. It was noted that the fim $H$ gene was detected in moderate and strong biofilm forming $E$. coli while absent in non biofilm isolates. The sequences showed $99 \%$ similarity with fim $H$ gene of $E$ coli.

CONCLUSION: The high binding ability of fim $\mathrm{H}$ could result in increased bacterial binding to target cells and increased pathogenicity of E.coli.

\section{INTRODUCTION}

Type 1 fimbriae are adhesion organelles expressed by many Gram negative bacteria. They facilitate adherence to mucosal surface and inflammatory cells as well as solid surfaces in vitro. This adherence results in biofilm formation on the surfaces thus contributing to the virulence of the strain as it leads to recurrent and drug resistant infections. E. coli is the most frequent pathogen responsible for up to $80 \%$ of UTIs

Uropathogenic E. coli (UPEC) have special virulence factors, including pilli or fimbriae, which mediate attachment to uroepithelial and vaginal cells, resistance to human serum bactericidal activity, haemolysin production and increased amount of $\mathrm{k}$ capsular antigen ${ }^{2}$. The bacterial attachment permits bacteria to resist mechanical elimination by the flow of urine thus increasing persistence of $E$. coli. UPEC also produce different types of adhesions, including type 1 fimbriae, which are essential for recognition and attachment to urinary tract receptors ${ }^{3}$. Among adhesions of UPEC, the adhesive subunit of type 1 fimbriae, fimH is a major determinant, which has high tropism for urinary tract receptor, thus, fimH adhesion is important in colonizing different niches of $E$. coli ${ }^{4}$. Surface attachment is mediated by fim $H$ adhesion, placed at the tip of the type 1 fimbriae, which prevents bacterial washout by urine flow and starts bacterial invasion ${ }^{5,6}$. Recurring UTIs increase the risk for pyelonephritis and long-term renal damage. The emergence of drugresistant uropathogens presents a further clinical challenge ${ }^{7}$. The present study was aimed for in vitro qualitative estimation of biofilm production in $E$ coli isolated from urinary tract infection and to correlate it with expression of Type 1 fimbriae by amplification of fimH gene by PCR using specific primers. The isolated genes were sequenced and compared.

\section{MATERIAL AND METHODS}

\section{Clinical isolates}

A total of 150 clean catch midstream urine samples were collected from patients in sterile containers visiting OPDs and IPDs of tertiary health care organization from Amravati district and immediately transported to the laboratory for further analysis.

\section{Quantitative Estimation of UTI}

A measured amount of urine i.e. $0.01 \mathrm{ml}$ was inoculated by using calibrated loop method on Hi-chrome UTI agar (HiMedia, India) and the plates were incubated at $37^{\circ} \mathrm{C}$ for $24 \mathrm{~h}$. After incubation colonies were counted. CFU $>10^{5}$ was considered as significant bacteriuria.

\section{Detection of biofilm formation by UPEC using Tissue} culture plate Method (TCP)

TCP method described by Christensen et al, 1995 is considered the Gold-standard method for biofilm detection [9]. Isolated UPEC were inoculated in $10 \mathrm{~mL}$ of trypticase soy broth with $1 \%$ glucose. Broths were incubated at $37^{\circ} \mathrm{C}$ for $24 \mathrm{~h}$. The cultures were then diluted 1:100 with fresh medium. Individual wells of sterile 96 well-flat bottom polystyrene tissue culture plates (Sigma-Aldrich, Costar, and USA) were filled with $200 \mu \mathrm{L}$ of the diluted cultures. The plates were incubated at $37^{\circ} \mathrm{C}$ for $24 \mathrm{~h}$. After incubation, contents of each well were removed by gentle tapping. The wells were washed with $0.2 \mathrm{~mL}$ of phosphate buffer saline ( $\mathrm{pH} 7.2)$ four times. This removed free floating bacteria. Biofilm formed by bacteria adherent to the wells were fixed by $2 \%$ sodium acetate and stained by crystal violet $(0.1 \%)$. Excess stain was removed by using deionized water and plates were kept for drying. Optical density (OD) of stained adherent biofilm was obtained by using ELISA reader at wavelength $570 \mathrm{~nm}$. OD value $<0.120$ was considered non biofilm forming, 0.120 0.240 was considered moderate and $>0.240$ as strong biofilm producing.

\section{DNA Extraction}

Three types of strains of UPEC i.e. strong, moderate and nonbiofilm forming were selected for DNA extraction. Genomic DNA templates for PCR amplification were gained from overnight growth of UPEC on Luria-Bertani broth (Hi Media, India). Bacterial culture (1.5 ml) was collected in eppendorff tube and centrifuged. Pellet was resuspended in $700 \mu l$ of cell lysis solution at room temperature, incubated for 5 minutes and centrifuged at $10,000 \mathrm{rpm}$ for 10 minutes. Supernatant $(500 \mu \mathrm{l})$ was collected in a fresh vial and $\mathrm{lml}$ of alcohol was added and mixed till DNA precipitated out. DAN was pelleted out by centrifuging and washed twice with $70 \%$ alcohol. DNA was dissolved in $100 \mu \mathrm{l}$ of TE buffer ( $\mathrm{pH} 8$ ).

Polymerase Chain Reaction

The isolated DNA from UPEC was subjected to screening for 
the presence of the fimH gene by PCR. The nucleotide sequence of the primers for amplification of the $\mathrm{fimH}$ gene is shown in Table 1.

Table - 1 Primers Used For Fimh Amplification

\begin{tabular}{|l|l|l|l|}
\hline Gene & Size (bp) & Description & Primer sequence (5'-3') \\
\hline fimH & $508 \mathrm{bp}$ & $\begin{array}{l}\text { Type l } \\
\text { fimbriae }\end{array}$ & $\begin{array}{l}\text { F-TTGAGAACGGAT } \\
\text { AAGCCGTGG } \\
\end{array}$ \\
& & & $\begin{array}{l}\text { R-GCAGTCACCTGC } \\
\text { CCTCCGGTA }\end{array}$ \\
\hline
\end{tabular}

PCR was performed in a $25 \mu \mathrm{l}$ reaction mixture containing $1.5 \mathrm{U}$ of Taq DNA polymerase, $2.5 \mu \mathrm{l}$ of $10 \times$ reaction buffer, $2 \mu \mathrm{l}$ of $2.5 \mathrm{mM}$ dNTP mixture, $10 \mathrm{pmol}$ of the forward and reverse primer and $0.5 \mu \mathrm{l}$ of DNA template. The PCR conditions were an initial denaturation at $94^{\circ} \mathrm{C}$ for 5 minute followed by 35 cycles of $94^{\circ} \mathrm{C}$ for $1 \mathrm{~min}, 58^{\circ} \mathrm{C}$ for 1 minute and $72^{\circ} \mathrm{C}$ for 1 minute and then a final extension at $72^{\circ} \mathrm{C}$ for 5 minute. PCR products were analyzed by agarose gel $(1 \%)$ electrophoresis using 200bp ladder.

\section{DNA Sequencing}

The PCR products were purified and sequenced bidirectionally by Sanger Dideoxy sequencing systems using ABI 3500 Genetic analyzer using ABI Big dye Tm terminator cycle sequencing kit at Central Instrumentation Centre at Sant Gadge Baba Amravati University, Amravati.

\section{RESULTS}

Quantitative estimation of urine samples

A total of 150 urine samples were analyzed during the period of study. Out of 150 urine samples, 98 samples had significant bacteriuria with the count more than $10^{5} \mathrm{cfu} / \mathrm{ml}, 40$ had insignificant growth and 12 samples no growth was observed.

\section{Bacterial isolates from urine samples}

Out of 98 urine samples with significant count, Escherichia coli was isolated in 77, Klebsiella pneumoniae in 10, Enterococcus faecalis in 9, Staphylococcus aureus and Micrococcus 1 in each sample (Fig l).

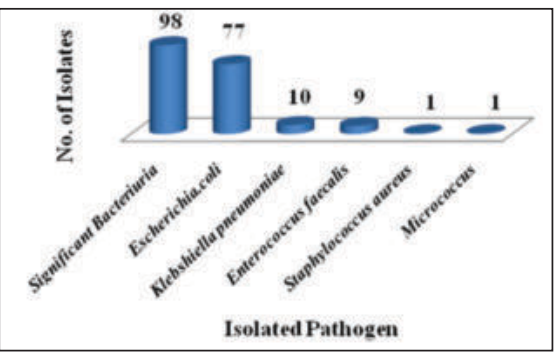

Fig 1.Bacterial isolates from urine sample Biofilm formation

Quantitative Tissue Culture Plate method of biofilm screening was done for 77 isolates of E. coli out of which 40 isolates were positive for biofilm formation (Fig 2).

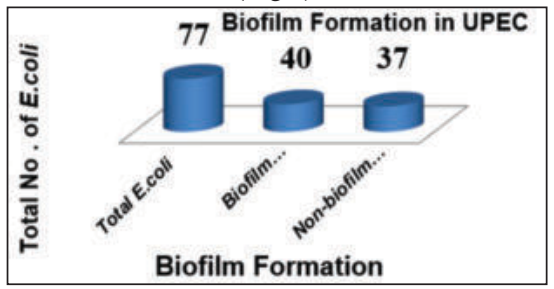

Fig 2. Number of biofilm forming and non-biofilm forming UPEC

They were categorized as strong, moderate and weak biofilm forming as explained in methodology. Wells with strong biofilm forming UPEC were stained dark as compared to wells with moderate and weak biofilm forming UPEC (Photo plate 1). Last horizontal and vertical rows were kept as controls containing only trypticase soy broth.

| www.worldwidejournals.com

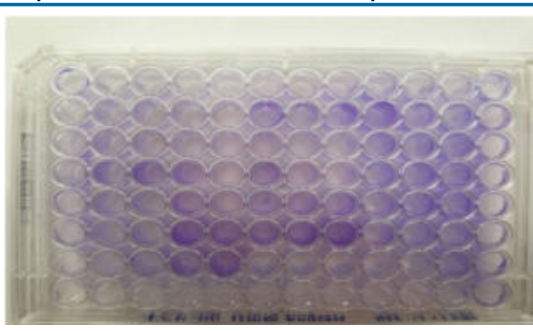

Photo plate 1: Tissue Culture Plate showing Biofilm formation by UPEC

Absorbance of the stained TCP was read by ELISA reader and the OD values $<0.120$ was considered as non biofilm forming, $0.120-0.240$ as moderate and $>0.240$ as strong biofilm producing. Greater the absorbance more is the density of the biofilm formed on the walls of TCP wells indicating enhanced adherence of UPEC. Thick biofilm will also contain more number of bacteria embedded within the biofilm matrix. Below graph represents comparative range of the OD values of strong, moderate and weakly adherent UPEC isolates. The adherence was noted as high as $0.57 \mathrm{~nm}$ indicating maximum biofilm formation (Fig 3).

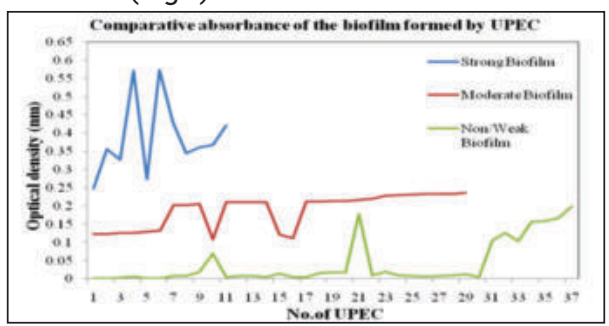

Fig 3: Absorbance of the biofilms formed by UPEC in the wells of tissue culture plate

Out of the total 40 biofilm forming UPEC, 11 isolates were classified as strong biofilm producing and 29 were moderate biofilm producing (Fig 4).

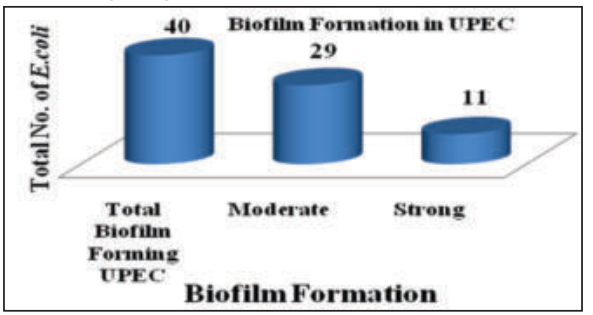

Fig 4. Total number of moderate and strong biofilm forming UPEC Polymerase Chain Reaction

The fimH gene was amplified using specific primers and appeared as a band of about $508 \mathrm{bp}$ on Agarose gel. The fimH gene was found in all the selected strong and moderate biofilm forming UPEC. But the gene was absent in the nonbiofilm forming isolates of UPEC (Photo plate 2).

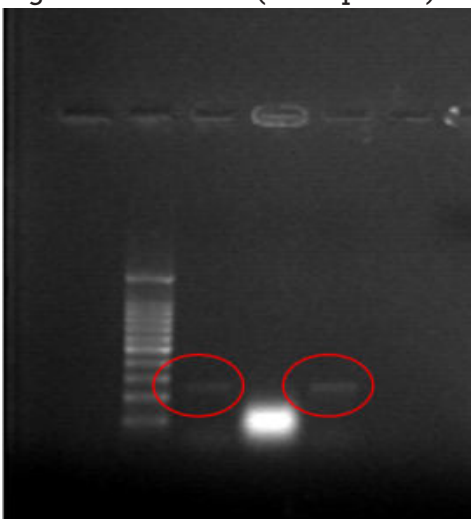

Photo plate 2: Polymerase Chain Reaction Amplification of the fim $H$ Gene 
I.ane 1- Tmpty

Lane 2- Ladder

\section{Lane3- Moderate biofilm forming UPEC I ane 4-Non biofilm forming UPTC Lane 5- Sirong biofilm forming UPEC}

\section{Sequencing}

Of total nine cultures, four strong biofilm forming and five weak biofilm forming strains were selected and the fim $\mathrm{H}$ gene was sequenced partially. The sequences were trimmed and blast search was conducted using nBlast. The sequences showed $99 \%$ similarity with fimH gene of $E$ coli. The sequences were aligned with $E$ coli strain k 12 sub strain MGI 655 's fimH sequence. The fimH gene sequences of all weak biofilm forming UPEC and the standard $E$ coli strain k 12 sub strain MGI 655's fimH sequence were 100\% similar. All strong biofilm UPEC fimH sequences were also similar. For final sequence alignment, two representative sequences of weak UPEC, one of strong UPEC and that of standard strain was used. The alignment is shown in figure 5. The major difference were found in strong biofilm strain of $E$ coli at base position number 108, where $\mathrm{T}$ was replaced by $\mathrm{C}$ and base position 177, where A present in weak biofilm forming strain was replaced by G. The sequences were submitted to NCBI. Accession numbers for weak biofilm forming strain is MZ456240 and for strong biofilm strain is MZ456241. The sequences were aligned using Bio Edit software ${ }^{21}$.

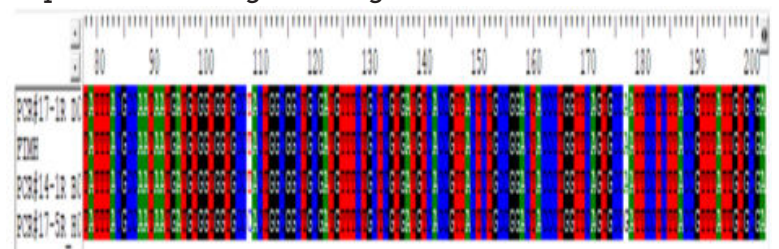

\#17-1RDO and \#14-1R BO:weak biofilm forming UPEC \#17-5R HO: strong biofilm forming UPEC FIMH: E coli strain k 12 sub strain MGI 655

Fig 5. Sequence alignment of fimH gene of weak and strong biofilm forming UPEC with standard strain of $E$ coli

\section{DISCUSSION}

Overall the study demonstrated that, E. coli is the most frequent isolate of urinary tract infection and have high propensity to form biofilm. From 150 urine samples, 98 had significant count out of which $78 \%$ isolates were E. coli.E. coli is the most common etiological agent involved in UTI, accounting for up to $80 \%$ of UTIs ${ }^{10}$. Bacterial adherence mediated by fimbriae is an essential prerequisite for colonization of the urinary tract. Uropathogenic Escherichia coli express a number of different adhesive organelles, including $\mathrm{P}$, type $1, \mathrm{~S}$, and FlC fimbriae ${ }^{11}$. Type 1 , or mannosesensitive, fimbriae are produced by $>80 \%$ of all uropathogenic E.coli.

In the present investigation, adherence of all UPEC was studied using quantitative Tissue Culture Plate method. It was found that, $11(14 \%)$ isolates displayed strong adherence, 29 $(37 \%)$ moderate and $37(48 \%)$ were weakly adherent to the walls of tissue culture plate. In the study conducted by Sharma et al (2009), the significant production of biofilm was $54 \%$ in uropathogenic E. coli. The study conducted by Matija et al (2009) demonstrated that $53 \%$ strains of UPEC were biofilm producing. In the present study the biofilm forming UPEC were $52 \%$.

The expression of type 1 fimbriae by $E$. coli is a virulence factor for pathogenesis of the urinary tract ${ }^{14,15}$ in the sense that they can produce a biofilm in within the urinary tract. The fimH protein is the receptor-recognizing element of type 1 fimbriae. FimH is located at the organelle tip in a short fibrillum and perhaps additionally intercalated along the fimbrial shaft. The components of the fimbrial organelle are encoded by the chromosomally located fim gene cluster. The fimH protein is produced as a precursor of 300 amino acids and is processed into a mature form of 279 amino acids. Fim $\mathrm{H}$ is exported, from the periplasm and across the outer membrane, is dependent on a fimbriae-specific export and assembly system constituted by the Fim C and FimD proteins ${ }^{11}$.

Expression of fimH gene of E. coli promotes adhesion, colonization and lead to increased rate of urinary tract infections. The onset of UTI results from the ability of UPEC attachment to urinary tract epithelial cells by specific adhesions including type 1 fimbriae [16]. The fim $H$ gene from all the UPEC isolates was amplified using specific primers. The gene was a band of about 508 bp on electrophoresis (fig 4). It was interesting to note that; fimH gene was present in all the 40 strong and moderate biofilm forming UPEC. But the gene was absent in the non-biofilm forming isolates of UPEC, thus demonstrating that fimH is required for the adherence of UPEC. Almost 52\% UPEC were positive for fimH gene.

In the study conducted by Hojati et al (2015) the fimH gene was found in 130 isolates $(92.8 \%)$ of the UPEC strains. Of 130 isolates positive for the fimH gene, $62(47.7 \%)$ and $68(52.3 \%)$ belonged to hospitalized patients and outpatients, respectively. The study indicated that more than $90 \%$ of $E$. coli isolates harbored the fimH gene. In the study conducted by Nam et al (2013), all virulence genes tested (including adhesins, iron acquisition, and protectin), except toxin genes, were detected among the canine UPEC isolates. Schembri et al (2000) reported that UTI fimH variants possess adaptations that enhance biofilm formation and suggests a novel role for fimH in UTIs associated with medical implants such as catheters. The study conducted by Krogfelt et al (1990), stated that type 1 fimbriae of Escherichia coli is surface organelle which mediate binding to D-mannosecontaining structures. Investigation of FimH gene occurrence was performed by Samah et al (2020) in $E$ coli isolated from clinical and environmental samples. The biofilm formation was detected in $66.6 \%$ strong, $33.30 \%$ moderate and weak biofilm producers not exist in clinical isolates, while $41 \%$ strong, $34.70 \%$ moderate and $8.60 \%$ for weak biofilm formation in environmental isolates. PCR assay of FimH showed that $95.23 \%$ were positive results for clinical isolates and $73.9 \%$ positive for environmental.

Uropathogenic $E$. coli had the higher occurrence rate for fimbriae gene $(\mathrm{FimH})$ compared with environmental isolates, stronger biofilm formation by clinical $E$. coli isolates than by environmental isolates. There was a significant correlation between biofilm formation and probability of FimH occurrence in both clinical and environmental isolates. Swaine et al studied the Positive selection identifies an in vivo role for FimH during urinary tract infection in addition to mannose binding. They performed in silico analysis of FimH gene sequences from $279 \mathrm{E}$. coli strains identified specific amino acids evolving under positive selection outside of its mannose-binding pocket. They found that mutating two of these residues (A27V/V163A) had no effect on phase variation, pilus assembly, or mannose binding in vitro. However, compared to wild-type, this double mutant strain exhibited a 10,000-fold reduction in mouse bladder colonization $24 \mathrm{~h}$ after inoculation and was unable to form IBCs even though it bound normally to mannosylated receptors in the urothelium. In contrast, the single A62S mutation altered phase variation, reducing the proportion of piliated cells, reduced mannose binding 8-fold, and decreased bladder colonization 30 -fold in vivo compared to wild-type. They concluded that positive selection analysis of FimH has separated mannose binding from in vivo fitness, suggesting that IBC formation is critical for successful infection of the mammalian bladder, providing support for more general use of in silico positive selection analysis to define the molecular 
underpinnings of bacterial pathogenesis.

\section{CONCLUSION}

We can conclude that type 1 fimbriae are present among biofilm forming UPEC to the extent that the fimH gene was detected in all strong and moderate biofilm forming UPEC and absent in non-biofilm forming UPEC. The high binding ability of fimH could result in increased bacterial binding to target cells and increased pathogenicity of $E$. coli. Thus fimH could be used to design vaccine for prevention of $E$. coli infections by blocking the bacterial attachment and colonization. In addition, fimH could be used as a tool for the extension of rapid detection-based assays as well as to differentiate between biofilm and non biofilm forming pathogens. Sequencing of the isolated fimH gene matched with $99 \%$ with $E$ coli fim $H$ gene and showed little difference in base position in strong and weak biofilm formation.

\section{Acknowledgement}

A research project entitled, "Evaluation of minimum inhibitory concentration and minimum biofilm eradicating concentration of selected antibiotics in biofilm producing uropathogenic E. coli", has been sanctioned by University Grants Commission, India to the author, Dr. Niraj Ghanwate. The above research is a part of this project. We highly acknowledge the University Grants Commission, New Delhi, India for its contribution.

\section{REFERENCES:}

[1] Wojnicz D (2007). Virulence factors of uropathogenic Escherichia coli strains isolated from children with chronic pyelonephritis.Adv Clin ExpMed;16(5):651 .

[2] Emody L, Kerenyi M and Nagy G (2003). Virulence factors of uropathogenic Escherichia coli.Int JAntimicrob Agents; 22(2):29-33.

[3] Oliveira FA, Paludo KS, Arend LN, Farah SM, Pedrosa FO and Souza EM (2011). Virulence characteristics and antimicrobial susceptibility of uropathogenic Escherichia coli strains. Genet Mol Res; 10(4):4114-25.

[4] Sokurenko EV, Chesnokova V, Dykhuizen DE, et al (1998). Pathogenic adaptation of Escherichia coli by natural variation of the FimH adhesin. Proc Natl Acad Sci USA 95:8922-6.

[5] Su C (2008). Female lower urinary tract infection.JTUA; 19:12-20.

[6] Finer G and Landau D (2004). Pathogenesis of urinary tract infections with normal female anatomy. Lancet Infec Dis; 4(10):631-5.

[1] Brown PD, Freeman A, and Foxman B (2002). Prevalence and predictors of trimethoprim-sulfamethoxazole resistance among uropathogenic Escherichia coli isolates in Michigan. Clin Infect Dis: 34: 1061-1066.

[8] Christensen GD, Simpson WA, Younger JA et al (1995). Adherence of coagulase negative Staphylococci to plastic tissue cultures: a quantitative model for the adherence of Staphylococci to medical devices. J Clin Microbiol;22:996-1006

[9] Mathur T, Singhal S, Upadhyay DJ, Fatma T and Batra A (2006). Detection of biofilm formation among the clinical isolates of Staphyllococci: An evaluation of three different screening methods. Indian Journal of Microbiology;24(1):25-27.

[10] Ronald A, (2003). The etiology of urinary tract infection: traditional and emerging pathogens. Dis Mon; 49:71-82.

[11] Klemm P (2004).Fimbriae:adhesion, genetics, biogenesis and vaccines. Boca Raton, FL: CRC Press.

[12] Sharma, M., Aparna, Yadav, S and Chaudhary, U. (2009), Biofilm production in uropathogenic E.coli.Indian Jof Pathology and Microbiol; 32 : (2).

[13] Matija Rijavec, Manca Müller-Premru, Breda Zakotnik and Darja gurBertok (2009), Virulence factors and biofilm production among Escherichia coli strains causing bacteraemia of urinary tract origin.Jof Med Microbiol.

[14] Connell H, Agace W, Klemm P, Schembri M, Mårild S (1996). Type 1 fimbrial expression enhances Escherichia coli virulence for the urinary tract. Proc Natl Acad Sci USA93:9827-32.

[15] Sokurenko EV, Feldgarden M, Trintchina E, Weissman SJ, Avagyan S and Chattopadhyay S (2004). Selection footprint in the FimH adhesin shows pathoadaptiveniche differentiation inEscherichia coli.Mol BiolEvol;21(7):1373-83.

[16] Kaczmarek A, Budzynska A and Gospodarek E (2012). Prevalence of genes encoding virulence factors among Escherichia coli with $\mathrm{Kl}$ antigen and nonKl E. coli strains. J Med Microbiol; 61 (10): 1360-1365.

[17] Hojati Z, Zamanzad B, Hashemzadeh M, Molaie R and Gholipour A, (2015). Detection of fimH gene in Uropathogenic Escherichia coli Strains Isolated from Patients with Urinary Tract Infection.Jundishapur JMicrobiol;8(2): 17520.

[18] Nam, Eui-Hwa, Ko S, Chae J, and Hwang C (2013). Characterization and Zoonotic Potential of Uropathogenic Escherichia coli Isolated from Dogs. J. Microbiol. Biotechnol;23(3): 422-429.

[19] Schembri MA, Sokurenko EV and Klemm P (2000). Functional Flexibility of the FimH Adhesin:Insights from a Random Mutant Library. Infection and Immunity; 62(5):2638-2646.

[20] Krogfelt KA, Bergman H, and Klemm P (1990). Direct Evidence that the fimH Protein Is the Mannose-Specific Adhesin of Escherichia coli Type 1 Fimbriae. Infection and Immunity; 58(6): 1995-1998.

[21] Hall T A (1999) BioEdit: user friendly Bioogical Sequence Alignment Analysis Programe forWindows 95/98/107. Nucleic acid symposium series. 41,95-98.

[22] Samah N Abd, Najeh H. Kadhum, and Hassan M. Abolmaali (2020) Investigation of Escherichia coli FimH gene occurrence isolated from clinical and environmental samples AIP Conference Proceedings 2290, 020045

[23] Swaine L Chen, Chia S Hung, Jerome S Pinkner, Jennifer NWalker, Corinne K Cusumano, Zhaoli Li, Julie Bouckaert, Jeffrey I Gordon, Scott J Hultgren
(2009)Positive selection identifies an in vivo role for FimH during urinary tract infection in addition to mannose binding. Proc Natl Acad Sci U S A. 106(52):22439-44 